\title{
Clinical Risk Factors Associated with Mortality Among Burn Patients Admitted to Jinnah Burn and Reconstructive Surgery Center, Lahore
}

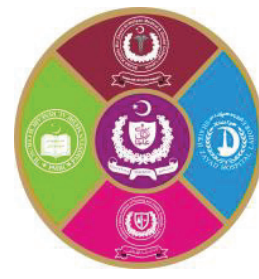

Fatima Sakhawat, Hamna Sohail, Fatima Zafar, Hassan Bashir, Hasan Shafique, Sana Iftikhar Department of Community Medicine, Allama Iqbal Medical College, Lahore

\begin{abstract}
Introduction: Burn injuries being highly devastating in nature, are associated with extensive morbidity and mortality. Although the incidence of burn injuries is less in developed countries but it is increasing in developing and low income countries. Aims \& Objectives: The aim of this study was to determine clinical risk factors associated with mortality among burn patients. Place and duration of study: Jinnah Burn and Reconstructive Surgery Center, Lahore from August 2018 till January 2019. Material \& Methods: Data was collected by researchers themselves by studying files of patients. Data analysis was done by using SPSS and Pearson Chi Square test was used to determine association of clinical risk factors with mortality among burn patients. Results: Out of 200 patients, 137 were males. The Mortality rate was $12.50 \%$. Cardiopulmonary arrest was the most common cause of death. Sepsis was the most common complication among burn patients. Higher mortality rates were observed among patients with arterial/venous and urinary catheterization done. Higher survival rates were seen in those patients who were treated with debridement, grafting surgeries, Colistin Use and aided by mechanical ventilation. Conclusion: Aid through mechanical ventilation, grafting surgeries, wound debridement, use of colistin are associated with improved survival outcomes among burn patients. Whereas inhalational injury, arterial/venous catheterization, urinary catheter and sepsis are seen as major predictors of mortality among burn patients.
\end{abstract}

Key words: mortality rate, burns, survival, clinical profiles

\section{INTRODUCTION}

A burn is defined as an injury to skin or other organic tissue primarily caused by heat radiation, electricity or contact with chemicals. ${ }^{1}$ Burns are the most common destructive form of injury. Improved outcomes after burn injuries are seen due to advances in field of medicine in terms of fluid replacement therapies, nutritional care pulmonary care, burn wound care and infection control practices. ${ }^{2}$ Much improvements in treatment strategies are still needed to further decrease the mortality associated with burn injury. According to facts and figures by WHO, burn injuries cause death of nearly 180,000 people per annum. ${ }^{3}$ Fire related burns is one of the major causes of disability adjusted life years (DALYS) in countries with average or below average income. ${ }^{4} \mathrm{WHO}$ statistics show that nearly $75 \%$ of burn injuries are common in South East Asia, Eastern Mediterranean and African subcontinent ${ }^{5}$ The incidence of burn injuries is estimated to be 243 per 100,000 people per year with mortality rate equal to $11.6 \%$ in low income countries of South East Asia in comparison to 1 death per 100,000 people in high income countries. ${ }^{6}$ A study of research paper from 14 African countries showed that Malawi has the highest mortality rate due to burn injury. ${ }^{7}$ Although there is no proper record keeping system functional in Pakistan, however, it is estimated that mortality due to burn injuries reaches up to $36 \%$ which is still 5 times higher than that in high income countries. People with severe burn injuries have increased risk of nosocomial infections which is considered to be the most common cause of death after burn. ${ }^{8}$ Patients who survive the burn injuries recover gradually, however recovery is incomplete in a large number of cases. Moreover it also has a remarkable effect on the psyche of patients as the patient has to undergo multiple reconstructive surgeries. ${ }^{9}$ Despite of the due importance, the epidemiological studies of burn injury are under-researched in Pakistan. ${ }^{10}$ By studying the co relation of outcome of burn injury with various clinical factors inpatients' profiles, this 
study may help in revealing predictors of mortality in burn patients.

\section{MATERIAL AND METHODS}

Lahore is a city of Punjab, Pakistan with a total population of 11.13 million. Jinnah burn and reconstructive surgery center is a part of Jinnah hospital, Lahore which is the second largest tertiary referral teaching hospital of the province of Punjab. The cross sectional study was conducted in Jinnah Burn and Reconstructive Surgery Center, Lahore over a period of 6 months (from August 2018 to January 2019). The permission for this study was obtained by Ethical Committee of the Institute. Informed consent was taken from patients. Confidentiality of the data of patients was maintained. Sample size was calculated using Open Epi Software at 95\% confidence interval with margin of error $5 \%$ and taking frequency of anticipated factor (mortality among burn patients) as $11.6 \%$. Calculated sample size was 137 . We recruited 200 patients in this study (total number of patients who were admitted during six months study period). Patients with contractures were excluded. Non Probability purposive sampling was done to recruit patients. A questionnaire was designed and pretested to collect the data about clinical profile of patients. Files of the patients were studied in detail by the researchers to obtain the dataon patients age, gender, inhalational injury, grafting, debridement surgeries, complications of burn injury, its outcomes, culture reports, venous catheterization, use of urinary catheter, support on mechanical ventilation and use of colistin.

\section{Statistical analysis:}

Data analysis was done by using Statistical Package for the social sciences (SPSS) version 24. Cross tabulation of data was done between outcome of burn injury and clinical profiles of the patients. We applied Pearson's chi square test to determine statistical significance of clinical risk factors with mortality among burn patients. $\mathrm{P}$ value $<0.05$ was considered statistically significant.

\section{RESULTS}

Out of $200,68.5 \%$ patients were males. Male to female ratio is $2.17: 1$. Majority $(35 \%)$ of the patients belonged to the age group of 16-25 years $(n=70)$. Majority $(52.5 \%)$ of the patients suffered from mixed thickness type of burn injury. (Graph-1) The mortality among burn patients was found to be $12.5 \%$. The most common complication of the burn injury was sepsis $42.6 \% \quad(\mathrm{n}=84)$, second most common complication seen was cardiopulmonary arrest $11.7 \%(\mathrm{n}=23)$. The most common cause of mortality seen among burn patients was cardiopulmonary arrest (92\%). Inhalation injury was present in $21.5 \%$ of the patients and mortality rate among them was $32.5 \%,(\mathrm{p}=0.00)$. Amputation of limb was done in only 5 percent of the patient. Wound culture was done in about $61 \%$ patients, about $21.5 \%$ of culture reports were negative. Among positive culture report the most common organism found was pseudomonas $18.85 \%$ followed by coinfection of Pseudomonas and Klebsiella $13.1 \%$ (Fig-1).Survival rate was statistically better among those patients in whom wound culture was done $(\mathrm{p}=0.00)$. Arterial/venous catheter was passed in about $79 \%$ of the patients. Mortality rate was higher among those in which arterial/venous catheter was passed $(96 \%, p=0.026)$. Urinary catheter was passed in about $64.5 \%$ patients. Mortality rate among patients who had urinary catheter passed was $88 \%$, higher than that of others who had not got urinary catheter passed $(\mathrm{p}=0.009)$. Only $16 \%$ patients required mechanical ventilation, survival rate was higher in those who were provided mechanical ventilation aid $(68.75 \%)$ as compared to those who weren't $(p=0.00)$. colistin was given in $29.5 \%$ patients and it improved survival rate $(\mathrm{p}=0.012)$ .Grafting surgeries were done in $36.5 \%$ patients and out of them only $2.73 \%$ patients deceased. So, grafting was found to improve the outcome of burn injury $(\mathrm{p}=0.007)$.Debridement was done in $35 \%$ patients. Out of 70 patients who were treated with debridement, $97.1 \%$ patients survived. So, like grafting debridement was also found to have increased the survival rates among the patients $(p=0.008)$. (Table- 1$)$

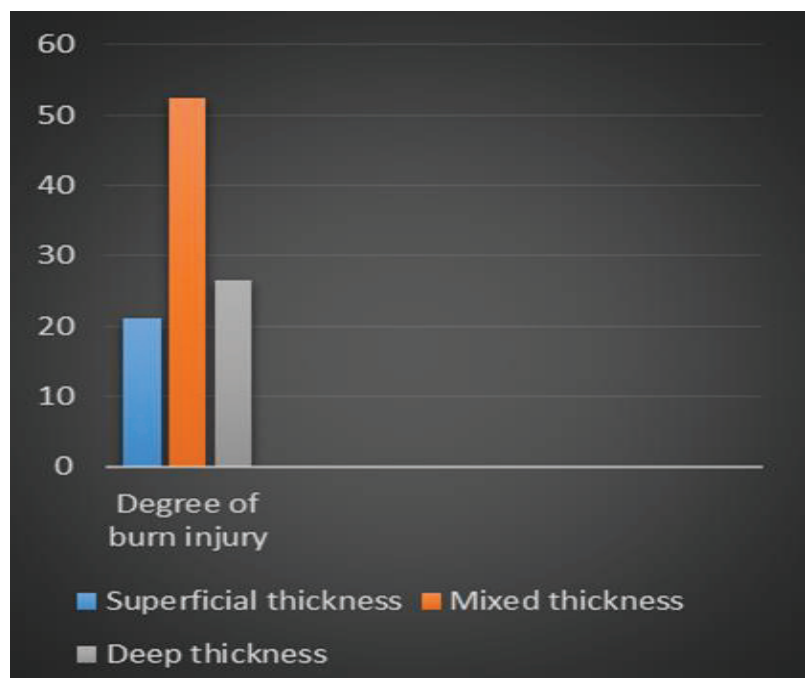

Graph-1: Degree of burn injury 


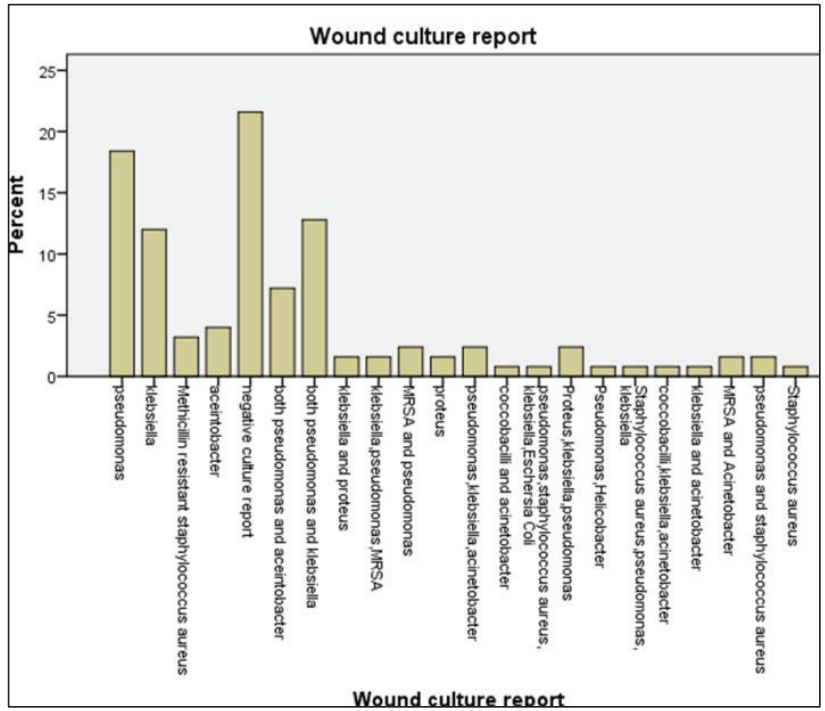

Figure 1: Wound culture report

\begin{tabular}{|c|c|c|c|c|}
\hline & \multicolumn{3}{|c|}{ Outcome of Burn Injury } & \multirow[b]{2}{*}{ P-value } \\
\hline & & $\begin{array}{c}\text { patient } \\
\text { deceased }\end{array}$ & $\begin{array}{c}\text { patient } \\
\text { survived }\end{array}$ & \\
\hline \multirow{2}{*}{$\begin{array}{l}\text { Inhalational } \\
\text { Injury }\end{array}$} & Yes & 14 & 29 & \multirow[t]{2}{*}{0.00} \\
\hline & No & 11 & 146 & \\
\hline \multirow{3}{*}{$\begin{array}{l}\text { Number of } \\
\text { debridements } \\
\text { done }\end{array}$} & 0 & 23 & 107 & \multirow[t]{3}{*}{0.008} \\
\hline & $1-2$ & 1 & 59 & \\
\hline & $>2$ & 1 & 9 & \\
\hline \multirow{3}{*}{$\begin{array}{l}\text { Number of } \\
\text { grafting } \\
\text { surgeries } \\
\text { done }\end{array}$} & 0 & 23 & 104 & \multirow[t]{3}{*}{0.007} \\
\hline & $1-2$ & 2 & 69 & \\
\hline & $>2$ & 0 & 2 & \\
\hline \multirow{2}{*}{$\begin{array}{l}\text { Venous } \\
\text { catheter } \\
\text { passed }\end{array}$} & Yes & 24 & 134 & \multirow[t]{2}{*}{0.026} \\
\hline & No & 1 & 41 & \\
\hline \multirow{2}{*}{$\begin{array}{l}\text { Urinary } \\
\text { catheter } \\
\text { passed }\end{array}$} & Yes & 22 & 107 & \multirow[t]{2}{*}{0.009} \\
\hline & No & 3 & 68 & \\
\hline \multirow{2}{*}{$\begin{array}{l}\text { Aid through } \\
\text { mechanical } \\
\text { ventilation }\end{array}$} & Yes & 10 & 22 & \multirow[t]{2}{*}{0.00} \\
\hline & No & 15 & 153 & \\
\hline \multirow[t]{2}{*}{ Use of colistin } & Yes & 2 & 57 & \multirow[t]{2}{*}{0.01} \\
\hline & No & 23 & 118 & \\
\hline \multirow{9}{*}{$\begin{array}{l}\text { Complications } \\
\text { of burn injury }\end{array}$} & Sepsis & 1 & 83 & \multirow[t]{9}{*}{0.00} \\
\hline & $\begin{array}{l}\text { Cardiopulmonary } \\
\text { arrest }\end{array}$ & 23 & 3 & \\
\hline & $\begin{array}{l}\text { Both sepsis and } \\
\text { cardiopulmonary } \\
\text { arrest }\end{array}$ & 0 & 2 & \\
\hline & Ectropion & 0 & 1 & \\
\hline & Shock & 0 & 1 & \\
\hline & Dry gangrene & 0 & 1 & \\
\hline & Respiratory arrest & 0 & 3 & \\
\hline & $\begin{array}{l}\text { Both sepsis and } \\
\text { respiratory } \\
\text { distress }\end{array}$ & 0 & 3 & \\
\hline & No complications & 1 & 78 & \\
\hline
\end{tabular}

Table-1: Relation of outcome of burn injury with variables

\section{DISCUSSION}

Finding out the outcome of burn injury and its association with various risk factors is important to improve not only management and care plan for our patients but to make better preventive schemes at individual and social levels in this era as well. The goal of the study is to guide health care professionals in improving approaches regarding treatment of the patient.

This study has determined the mortality rate as $12.5 \%$ among burn patients which is far less than that of other studies done. Another study from Karachi Pakistan had shown mortality rates of $36.12 \%$ in that region due to the same cause. ${ }^{11} \mathrm{~A}$ Malaysian study had shown the mortality rates of $12.2 \%$ among burn patients which is closer to our study. ${ }^{12}$ A multi institutional study form United States had shown mortality rate of $3.3 \%$ due to burn injury ${ }^{13}$ which can be attributed to good Infection control practices over there. Despite the excel in disease management and patient care, Pakistan is still facing higher mortality rates due to burn injury in comparison to developed countries in the western world which implies that much is needed to be done practically to lower the mortality in our patients. The various predictors of mortality in burn patients include inhalational injury, arterial/venous catheterization, urinary catheterization, complications such as sepsis and cardiopulmonary arrest, aid through mechanical ventilation, grafting surgeries and debridement of the wound. A generalized improved impact of use of colistin on outcome of burn injury was also observed.

It has been observed that in all the cases of mortality, the cause was cardiopulmonary arrest, except two cases; out of them, the cause of death of one patient was declared as septic shock and the cause of death of the other patient was unknown. Burn injury causes surge of catecholamines which causes cardiac stress after burn injury. This is because of increased myocardial oxygen utilization which causes myocardial hypoxia and cardiac death. ${ }^{14}$

Most common complication among those who have survived was nosocomial infections/sepsis. About $47.51 \%$ patients suffered from such complications in our hospital settings. Sepsis triggers inflammatory cascades which causes multi organ damage and failure leading to death of the patient. ${ }^{15}$ According to our study, most of the patients who suffered from inhalational injury, died. Inhalational injury is associated with exposure of respiratory tract to heat leading to thermal burn inside and 
damage due to constitutional toxins of inhaled smoke. ${ }^{16}$

Survival rates were found to be high among those who were aided by mechanical ventilation than those who were not. This is because of the reason that burn patients suffer from respiratory distress, especially when it comes to diagnose the cases of inhalational injury associated with burn trauma .In most of the cases, the culture report was positive for Pseudomonas and second most frequent organism found infecting was Klebsiella. Survival rates was high in patients who had negative microbe culture report. Out of the patients who died, most had urinary foley catheter and IV line passed. Although IV lines are very important in administering medicines such as saline and ringer's lactate and benefits of foleycathetar can also never be denied when it comes to drain the urine and treat exaggerated urinary continence but both of them are associated with increased risk of infections due to iatrogenic causes. This may be due to flora at periuretheral area and skin and also the biofilm formation. This bacterial infection when spreads through the blood is a cause of multi organ dysfuntion and consequently death. Yet, the primary factors are always the total body surface area burnt, degree and site of burn injury. ${ }^{17}$ Use of IV colistin is highly protective in patients of burn injury according to our study. It is a polymyxin $\mathrm{E}$ antibiotic very effective against multidrug resistant strains of pseudomonas and aceintobacter species. ${ }^{18}$ Debridement of a wound was clearly found as a beneficial factor to achieve the health and well being in a patient. Burn patients lack primary protective layers of the body such as skin and fascia which expose underlying tissue to microorganisms in the environment and surrounding thus increasing the risk of infections. However, some time these infections being destructive, damage the tissue to such an extent that it cannot be spared by using antibiotics and the only preventive treatment left to prevent the spread of infections is to do debridement and grafting. In severe cases amputation of the limb may be the option to save the life from vicious septic cycles and multi organ failures. ${ }^{19}$

This study highlights the importance of certain factors towards reducing the burden of mortality due to burn injury, however, a lot of research with larger sample sizes is still needed to support our findings. Moreover, mortality rate in our study is low in comparison to other studies in Pakistan, this may be due to the reason that this study was conducted at tertiary care hospital. Therefore, to get a more generalized result, such studies should also be promoted at level of District and Tehsil Headquarter
Hospital settings so that a significant addition can be made in terms of exploring the causes and risks of mortality among burn patients. Attempt should be made to find out the ways which can help in further reduction of the mortality rates taking into account the impact of associated risk factors and to develop the strategies for improvement of patient management and health care services accordingly.

\section{CONCLUSION}

Aid through mechanical ventilation, grafting surgeries, wound debridement, use of colistin are associated with improved survival outcomes among burn patients. Whereas Inhalational injury, arterial/venous catheterization, urinary catheter and complications such as cardiopulmonary arrest and sepsis are seen as major predictors of mortality among burn patients.

\section{REFERENCES}

1. Mathers C, Fat DM, Boerma J. The global burden of disease: 2004 update. BMC Res Notes, 6 (2013), p. 545 Geneva: World Health Organization; 2008.

2. Hussain A, Choukairi F, Dunn K. Predicting survival in thermal injury: A systematic review of methodology of composite prediction models. Burns. 2013 Feb 2; Epub ahead of print. [PubMed]

3. Global Burden of Disease. The Institute for Health Metrics and Evaluation. Geneva: World Health Organization; 2008.

4. Peck MD. Epidemiology of burns throughout the world. Part I: Distribution and risk factors. Burns. 2011; 37(7):1087-1100. [PubMed]

5. WHO fact sheets;

http://www.who.int/mediacentre/factsheets/fs36 5/en/

6. Global Burden of Disease. The Institute for Health Metrics and Evaluation. http://www.healthdata.org/gbd

7. A. Golshan, C. Patel, A.A. Hyder A systematic review of the epidemiology of unintentional burn injuries in South Asia J Public Health (Oxf), 35 (3) (2013), pp. 384-396.

8. O'Keefe GE, Hunt JL, Purdue GF. An evaluation of risk factors for mortality after burn trauma and the identification of genderdependent differences in outcomes. J Am Coll Surg. 2001; 192(2):153-60. [PubMed]

9. Rafla K. Tredget EE. Infection control in burn unit. Burn 200; 37:5-15. 
10. Batra AK. Burn Mortality: Recent trends and socio cultural determinants in rural India Burn. PUBMED. 2003; 29:270-5.

11. Ibran et al. Mortality associated with burn injury- across sectional study from Karachi, Pakistan. BMC Research Notes 2013; 6:545.

12. Tan Chor Lip, Tan, Thomas, Imran, Azmah Tuan Mat: Survival analysis and mortality predictors of hospitalized severe burn victims in a Malaysian burns intensive care unit. 2019 Jan.

13. Zavlin, Chegireddy, Boukovalas, Nia, Branski, Friedman, Echo. Multi-institutional analysis of independent predictors for burn mortality in the United States: 2018 Aug 22; 6:24.

14. Felicia N Williams, MD; David N Herndon, MD;Oscar E Suman, PhD, ; Jong O Lee, MD; William B Norbury, MD; Ludwik K Branski, MD; Ronald P Mlcak, PhD; and Marc G Jeschke, MD, PhD1: Changes in cardiac physiology after severe burn injury: J Burn Care Res. 2011 Mar-Apr.

15. Bharwana, S., Farid, M., Ali, S., Rizwan, M., Nadeem, M., Haider, M., Tauqeer, H., SallahUd-Din, R., \& Ahmad, R. (2016). Mortality rate associated with hospital acquired infections among burn patients. Biomedical Research and Therapy, 3(9), 790-799.

16. Vittorio Pavoni, Lara Gianesello, Laura Paparella, Laura Tadini Buoninsegni, and Elisabetta Barboni: Outcome predictors and quality of life of severe burn patients admitted to intensive care unit: 2010 Apr 27.

17. Dr. Barbara W. Trautner, MD and Dr. Rabih O. Darouiche, MD Catheter-Associated Infections Pathogenesis Affects Prevention.

18. Ludwik K. Branski, Ahmed Al-Mousawi, Haidy Rivero, Marc G. Jeschke, Arthur P. Sanford, and David N. Herndon Emerging Infections in Burns: Surg Infect (Larchmt). 2009 Oct; 10(5): 389-397.

19. Bowo SA, Soedjana H. Effectiveness of Early Excisional Debridement in Burn Injuries to Sepsis Incidence and Mortality rate at Burn Unit of Hasan Sadikin Hospital. J Plast Rekons. 2017, Jan 24; 3(2):40-4

\section{The Authors:}

Fatima Sakhawat

$5^{\text {th }}$ year M.B.B.S. student,

Department of Community Medicine,

Allama Iqbal Medical College, Lahore.

HamnaSohail

$5^{\text {th }}$ year M.B.B.S. student,

Department of Community Medicine,

Allama Iqbal Medical College, Lahore.

Fatima Zafar

$5^{\text {th }}$ year M.B.B.S. student,

Department of Community Medicine,

Allama Iqbal Medical College, Lahore.

Hassan Bashir

5th year M.B.B.S. student,

Department of Community Medicine,

Allama Iqbal Medical College, Lahore

Hasan Shafique

$5^{\text {th }}$ year M.B.B.S. student,

Department of Community Medicine,

Allama Iqbal Medical College, Lahore.

Dr. Sana Iftikhar

Assistant Professor,

Department of Community Medicine,

Allama Iqbal Medical College, Lahore.

\section{Corresponding Author:}

Fatima Sakhawat

$5^{\text {th }}$ year M.B.B.S. student,

Department of Community Medicine,

Allama Iqbal Medical College, Lahore.

E-mail: fatimasakhawat1@gmail.com 